\title{
Gesetzgebungskompetenz für den Strafvollzug - Föderalismuskommission wünscht Übertragung auf Länder
}

Heinz Cornel

m Dezember 2004 wurde bekannt, dass die Kommission zur Modernisierung der bundesstaatlichen Ordnung (kurz: Föderalismuskommission) unter dem gemeinsamen Vorsitz von Franz Müntefering (MdB) und Ministerpräsident Dr. Edmund Stoiber beabsichtigte, die Gesetzgebungskompetenz für den Strafvollzug, einschließlich U-Haft und Jugendstrafvollzug, vom Bund auf die Länder zu übertragen. Eine inhaltliche Debatte über dieses Vorhaben hatte vorher nicht stattgefunden und in der Öffentlichkeit wurde das zunächst kaum bemerkt. Viel spricht dafür, dass diese Aufgabe der einheitlichen bundesdeutschen Mindeststandards in diesem hochsensiblen Bereich der Eingriffe in Grundrechte sich fachlich nicht begründen lässt, sondern nur Sparpotentiale eröffnen und den Abschied von den Zielen des Strafvollzugsgesetzes einleiten soll. Als Tauschobjekt eignete sich dieser Themenbereich wohl, weil keine gar zu große Zahl von Fürsprechern für diese Zielgruppe der Kriminalpolitik erwartet wurde.

Diese Pläne waren Anlass, den beiliegenden Aufruf zu verfassen, ihn an Kollegen und Kolleginnen zu senden und um Unterstützung $\mathrm{zu}$ bitten. Innerhalb weniger Tage gingen daraufhin nicht nur mehr als 80 unterstützende emails und Faxe sowie viele Anfragen bei mir ein und die Presse berichtete darüber- oft mit zustimmenden Kommentaren (Die Zeit sprach am 16.12.2004 von einem »Wettbewerb der Schäbigkeit) - mehrfach, sondern auch zahlreiche Verbände hatten sich gegen diese Verlagerung der Gesetzgebungskompetenz ausgesprochen. Genannt seien hier die Deutsche Vereinigung für Jugendgerichte und Jugendgerichtshilfen, die Bundesvereinigung der Anstaltsleiter im Strafvollzug, der Bund der Strafvollzugsbediensteten Deutschlands, die Vereinigung der Hessischen Strafverteidiger, die Katholische Bun-
des-Arbeitsgemeinschaft Straffälligenhilfe im Deutschen Caritasverband und die Evangelische Kirche in Deutschland, vertreten durch Bischof Dr.Wolfgang Huber.

Erstaunt kann man sein, dass auch die Bundesjustizministerin diesem Vorschlag der Föderalismuskommission zustimmte, obwohl sie sich noch im Sommer im Vorwort zu den Empfehlungen des Europarats »Freiheitsentzug « sehr für die »Schaffung und den Erhalt einheitlicher Mindeststandards im Justizvollzug « eingesetzt hatte, weil dies notwendig sei »in diesem sensiblen Bereich, der den betroffenen Menschen das größte Rechtsgut, ihre Freiheit, nimmt und daher den Rechtsstaat hochgradig fordert...". Mit Hinblick auf die europäische Ebene lobte sie die »Vereinheitlichung und Vergleichbarkeit nicht nur der Rechtsgrundlagen des Justizvollzuges, sondern auch der praktischen Ausgestaltung.« Da möchte man nicht nur zustimmen, sondern auch hoffen, dass diese Einsicht die zukünftige Stellungnahme des Bundesjustizministeriums zur Verlagerung der Gesetzgebungskompetenz bestimmt, denn trotz des Scheiterns der Föderalismuskommission Mitte Dezember 2004 ist das Thema keinesfalls vom Tisch.

Im Folgenden werden der Aufruf und die Liste der Unterzeichner abgedruckt:

\section{Gesetzgebungskompetenz für den Strafvollzug muss beim} Bund bleiben Dezember 2004

Strafrechtswissenschaftler, Strafvollzugsrechtler und Kriminologen sprechen sich gegen die Änderungsvorschläge der Föderalismuskommission aus.

Mehr als 100 Jahre musste Deutschland nach seinem Strafge- setzbuch und der Strafprozessordnung auf ein einheitliches Strafvollzugsgesetz warten, das 1976 mit den Stimmen aller Parteien nach jahrzehntelanger Diskussion verabschiedet wurde. Diese Rechtseinheit innerhalb Deutschlands, aber auch die systematisch sachlich gebotene Einheit von materiellem Recht, Verfahrens- und Vollzugsrecht soll nun aufgelöst werden. Das wird negative Folgen haben - nicht zuletzt für die Qualität des Strafvollzugs, die Verwirklichung des Vollzugsziels der Resozialisierung und damit des Rückfallrisikos.

Das Strafvollzugsrecht betrifft einen Kernbereich staatlicher Tätigkeit, im Rahmen derer die intensivsten Eingriffe in die Rechte von verurteilten Bürgern stattfinden. Diese Eingriffe müssen grundsätzlich gleichermaßen ausgestaltet werden und dürfen nicht zur Disposition unterschiedlicher und wechselnder landespolitischer Orientierungen gestellt werden. Der unsere Verfassung prägende Grundsatz, dass in den Ländern möglichst einheitliche Lebensverhältnisse herzustellen sind, muss im Strafvollzug in besonderem Maße Beachtung finden. Es war erklärtes Ziel der mehr als 100-jährigen Gesetzgebungsarbeit, die 1976 zur Verabschiedung des Strafvollzugsgesetzes führte, der Auseinanderentwicklung der Lebensverhältnisse in den einzelnen Bundesländern entgegen $\mathrm{zu}$ wirken. Deshalb wurden, u.a. zeitgleich mit dem StVollzG, bundeseinheitliche Verwaltungsvorschriften erlassen.

Die Auflösung der Rechtseinheit im Strafvollzugsrecht würde die schon jetzt erhebliche Ungleichheit der Lebensverhältnisse in Bereichen der Resozialisierung und insbesondere der Entlassungsvorbereitung (offener Vollzug, Vollzugslockerungen etc.) noch weiter vertiefen. Es besteht die Gefahr, dass einzelne Bundesländer den Strafvollzug auf einen reinen Verwahrvollzug reduzieren und die für eine erfolgreiche Resozialisierung notwendigen personellen und sachlichen Mittel weiter kürzen, während andere das alleinige Ziel der Resozialisierung und damit der Verhinderung weiterer Kriminalitätsopfer ernst nehmen.

Wie weit wäre der Strafvollzug in den neuen Bundesländern heute ohne einheitliches Strafvollzugsgesetz und wäre es wirklich wünschenswert, dass Mindeststandards durch Vereinbarungen von 16 Justizverwaltungen und Entscheidungen der Bundesgerichte erzielt werden? Soll das Jugendstrafvollzugsgesetz nach 30 Jahren Reformdiskussion wiederum kurz vor der Einigung gestoppt werden?

Letztlich geht es darum, ob man bei so erheblichen Grundrechtsbeschränkungen, wie sie der Strafvollzug mit sich bringt, gleiche Rechte und Pflichten für alle Gefangenen möchte, die aufgrund gleicher Strafgesetze verurteilt wurden oder ob man die jeweiligen Ziele und Standards, das Ausstattungsniveau und die Sicherheit den wechselnden Mehrheiten in den Landtagen überlassen will, wobei man zusätzlich befürchten müsste, dass in den Wahlkämpfen vollzugspolitische Themen aus taktischen Überlegungen abgehandelt werden. Das gab es zwar auch in der Vergangenheit und auch auf Bundesebene - das würde sich aber sicher erheblich verstärken.

Sachlich tragfähige Argumente werden für die Auflösung der Rechtseinheit nicht genannt. Es hat den Anschein, dass das Thema Strafvollzug zwischen Bund und Ländern nur Verhandlungsmasse ist, um sich bei anderen Regelungsbereichen durchzusetzen.

Wissenschaft, Praxis und Politik waren sich in den letzten 25 Jahren häufig nicht einig über die Bewertung einzelner Regelungen des Strafvollzugsgesetzes und deren Umsetzung - aber die größere Rechtssicherheit und die bundesdeutsche Rechtseinheit wurden regelmäßig von allen befürwortet und sind ein hohes Gut, das nicht grundlos aufgegeben werden darf. 